\title{
Is cardiothoracic point-of-care ultrasonography the future of heart failure diagnosis?
}

\author{
Colin Bell MD, Heather Murray MD MSc, Paul Atkinson MB BCh BAO MA
}

- Cite as: CMAJ 2021 November 8;193:E1702-3. doi: 10.1503/cmaj.211763

See related article at www.cmaj.ca/lookup/doi/10.1503/cmaj.210083

M

ore than 5 of every 1000 people in Canada will receive a new diagnosis of heart failure this year. ${ }^{1}$ Many more will have respiratory symptoms that will prompt the ordering of chest radiography. ${ }^{2,3}$ In a related research article, Torres and colleagues challenged an established rule-of-thumb of chest radiography, namely that a cardiac silhouette wider than half of the chest diameter represents cardiomegaly, which is suggestive of underlying heart failure. ${ }^{3}$ They compared cardiothoracic ratio measurements on chest radiographs with the gold standard of assessing cardiac enlargement with magnetic resonance imaging (MRI), ${ }^{3}$ and showed that the positive and negative likelihood ratios (LR) of the cardiothoracic ratio are unhelpful in either confirming or refuting cardiomegaly at any cutpoint. The absolute size of the cardiac silhouette was more useful, with a maximum heart diameter greater than $19 \mathrm{~cm}$ for men and greater than $13 \mathrm{~cm}$ for women associated with clinically useful LRs for predicting cardiac enlargement. ${ }^{3}$

The symptoms of heart failure are nonspecific and overlap with symptoms of many other life-threatening and benign conditions. Thus, a diagnosis of heart failure is rarely pursued in isolation and can be challenging to make or to rule out. Paroxysmal nocturnal dyspnea, a symptom clinicians strongly associate with heart failure, has been shown to have limited usefulness in the diagnosis of heart failure, with a positive LR of 2.2 and a negative LR of $0.7{ }^{2}$ The clinical examination is no better. A third heart sound is only marginally useful (positive LR 1.14 , negative LR 0.08 ) and basal crackles on chest auscultation even less so (positive LR 1.95, negative LR 0.37). ${ }^{2}$ The elevated jugular venous pressure also contributes minimally to making the diagnosis (positive LR 1.06, negative LR 0.62). ${ }^{2}$

Laboratory testing for brain natriuretic peptide (BNP) and $\mathrm{N}$-terminal pro BNP (NT-proBNP) has been recognized as effective for diagnosing heart failure. Levels of BNP and NT-proBNP below $100 \mathrm{pg} / \mathrm{mL}$ (negative LR 0.30 ) and $300 \mathrm{pg} / \mathrm{mL}$ (negative LR 0.01), respectively, dramatically reduce the probability of heart failure, and values above $500 \mathrm{pg} / \mathrm{mL}$ (positive LR 70) and $1800 \mathrm{pg} / \mathrm{mL}$ (positive LR 6.6), respectively, largely confirm the

\section{Key points}

- Heart failure is a common disease that remains challenging to diagnose.

- Chest radiography is of limited utility in the diagnosis or exclusion of heart failure as a cause of shortness of breath.

- Cardiothoracic point-of-care ultrasonography (POCUS) can be used to diagnose heart failure reliably and may identify its underlying cause.

- Barriers to the use of POCUS are diminishing, making it a promising tool for diagnosis in patients with suspected heart failure.

diagnosis. ${ }^{2}$ However, many patients will fall into the intervening ranges and diagnostic uncertainty will persist, especially given that other conditions can lead to elevated BNP and NT-proBNP.

A recent systematic review of studies that compared the diagnostic accuracy of cardiothoracic point-of-care ultrasonography (POCUS) with chest radiography in adults with symptoms suggestive of acute decompensated heart failure found that chest radiography performs poorly, with a sensitivity between 0.70 and $0.76 .{ }^{4}$ The findings of the related study ${ }^{3}$ would seem to support a growing understanding that chest radiography is of limited use in establishing or ruling out heart failure.

Consultative (formal) echocardiography has long been the gold standard test for heart failure, as it both confirms the diagnosis and establishes the cause. ${ }^{2}$ However, wait times for consultative echocardiography can be long, which delays diagnosis and initiation of therapy. Point-of-care ultrasonography, performed by a trained front-line clinician at the bedside, uses the same technology as echocardiography. A blinded crosssectional study of physicians with variable POCUS training showed that noncardiologists can accurately diagnose common causes of heart failure using POCUS. ${ }^{5}$

The aforementioned 2019 systematic review found that using POCUS to identify the presence and distribution of B-line artifacts 
(indicating lung interstitial fluid) was clinically useful in confirming a diagnosis of heart failure (positive LR 8.8, negative LR 0.13). ${ }^{4}$ A 2015 observational study directly compared the commonly used composite diagnostic approach (i.e., clinical examination, serum NT-proBNP and chest radiography) with cardiopulmonary POCUS alone (i.e., without clinical examination) among patients who presented with acute dyspnea. ${ }^{6}$ Cardiopulmonary POCUS was more accurate for diagnosing acute left-sided heart failure than the composite approach, and it took only about 12 minutes to perform during a single bedside assessment. ${ }^{6}$ The added advantage of cardiopulmonary POCUS is that clinicians can often establish a cause for heart failure at the time of diagnosis. Patients' trust in their clinician may also be enhanced when they use POCUS. ${ }^{7}$

What does this mean for our approach to the evaluation of patients with suspected heart failure in primary and emergency care in the future? Physicians are increasingly being trained to perform POCUS and most medical schools now educate learners about POCUS, often linking it with anatomy courses, offering live visualization of dynamic anatomic relationships and the anatomic variability present in humans. ${ }^{8}$ Recent studies that evaluated training found that learners can become competent in POCUS relatively quickly, ${ }^{9,10}$ and that physicians and paramedics maintain their competency even if they use the skill infrequently at the bedside. ${ }^{9,11}$

Remote- and artificial intelligence-guided POCUS technology is already commercially available for use in settings where clinicians lack training or require additional decision support. ${ }^{12}$ Moreover, artificial intelligence technology is increasingly integrated in commercially available POCUS devices, which allows, for example, automated ejection fraction and B-line quantification; ${ }^{13}$ however, this rapidly developing technology requires careful validation. Given the increasing affordability of devices, POCUS may soon be more globally accessible than radiography.

Critics may argue that, since POCUS is performed at the bedside, maintaining quality control and optimal record-keeping is a challenge. However, POCUS machines can be linked to hospital picture archiving and communications systems, or commercially available software can archive POCUS images in an easily searchable manner integrated with the electronic medical record, and can deidentify images, as required.

Although ordering chest radiography may be helpful in some clinical circumstances, the test's diagnostic utility is limited for patients with suspected heart failure. Emerging evidence suggests that the time has come for us to embrace cardiothoracic POCUS as routine.

\section{References}

1. Heart disease in Canada. Ottawa: Public Health Agency of Canada; 2017.

2. Inamdar AA, Inamdar AC. Heart failure: diagnosis, management and utilization. J Clin Med 2016;5:62.

3. Torres FS, Eifer DA, Times FS, et al. Diagnostic performance of chest radiography measurements for the assessment of cardiac chamber enlargement. CMAJ 2021;193:E1683-92.
4. Maw AM, Hassanin A, Ho PM, et al. Diagnostic accuracy of point-of-care lung ultrasonography and chest radiography in adults with symptoms suggestive of acute decompensated heart failure: a systematic review and meta-analysis. JAMA Netw Open 2019;2:e190703.

5. Adhikari S, Fiorello A, Stolz L, et al. Ability of emergency physicians with advanced echocardiographic experience at a single center to identify complex echocardiographic abnormalities. Am J Emerg Med 2014;32:363-6.

6. Gallard E, Redonnet J-P, Bourcier J-E, et al. Diagnostic performance of cardiopulmonary ultrasound performed by the emergency physician in the management of acute dyspnea. Am J Emerg Med 2015;33:352-8.

7. Howard ZD, Noble VE, Marill KA, et al. Bedside ultrasound maximizes patient satisfaction. J Emerg Med 2014;46:46-53.

8. Ma IWY, Steinmetz P, Weerdenburg K, et al. The Canadian medical student ultrasound curriculum: a statement from the canadian ultrasound consensus for undergraduate medical education group. J Ultrasound Med 2020; 39:1279-87.

9. Ackil DJ, Toney A, Good R, et al. Use of hand-motion analysis to assess competence and skill decay for cardiac and lung point-of-care ultrasound. AEM Educ Train 2020;5:e10560.

10. Duanmu Y, Henwood PC, Takhar SS, et al. Correlation of OSCE performance and point-of-care ultrasound scan numbers among a cohort of emergency medicine residents. Ultrasound J 2019;11:3.

11. Schott CK, LoPresti CM, Boyd JS, et al. Retention of point-of-care ultrasound skills among practicing physicians: findings of the VA National POCUS Training Program. Am J Med 2021;134:391-9.e8.

12. Olivieri PP, Verceles AC, Hurley JM, et al. A pilot study of ultrasonographynaive operators' ability to use tele-ultrasonography to assess the heart and lung. J Intensive Care Med 2020;35:672-8.

13. Shokoohi H, LeSaux MA, Roohani YH, et al. Enhanced point-of-care ultrasound applications by integrating automated feature-learning systems using deep learning. J Ultrasound Med 2019;38:1887-97.

Competing interests: Paul Atkinson reports royalties from textbooks on the topic of ultrasonography in emergency medicine, and honoraria for teaching with CanPOCUS.com. No other competing interests were declared.

This article was solicited and has not been peer reviewed.

Affiliations: Department of Emergency Medicine (Bell), Cumming School of Medicine, University of Calgary, Calgary, Alta.; Department of Emergency Medicine (Murray), Faculty of Health Sciences, Queen's University; Emergency Medicine (Murray), Kingston Health Sciences Centre, Kingston, Ont.; Department of Emergency Medicine (Atkinson), Dalhousie University, Saint John Regional Hospital; Dalhousie Medicine New Brunswick (Atkinson), Saint John, NB

Contributors: All of the authors contributed to the conception and design of the work, drafted the manuscript, revised it critically for important intellectual content, gave final approval of the version to be published and agreed to be accountable for all aspects of the work.

Content licence: This is an Open Access article distributed in accordance with the terms of the Creative Commons Attribution (CC BY-NC-ND 4.0) licence, which permits use, distribution and reproduction in any medium, provided that the original publication is properly cited, the use is noncommercial (i.e., research or educational use), and no modifications or adaptations are made. See: https://creativecommons.org/licenses/by-nc-nd/4.0/

Disclaimer: Heather Murray is an associate editor for CMAJ and was not involved in the editorial decision-making process for this article.

Correspondence to: Colin Bell, colinbell85@gmail.com 